\title{
Some Extensions of Kannan Fixed Point Theorem on 2-Metric Spaces
}

\author{
Kirty Chauhan ${ }^{1}$, G. P. S. Rathore ${ }^{2}$, Bijendra Singh $^{3}$ \\ ${ }^{1}$ Department of Mathematics, A T C, Indore, India, \\ ${ }^{2}$ College of Horticulture, Mandsaur, India \\ ${ }^{3}$ S.S. in Mathematics, Vikram University, Ujjain, India.
}

\begin{abstract}
In this paper we established fixed point theorems in 2-Metric Spaces by using some new extensions of Kannan fixed point theorem obtained by Kannan [1,2]. The result can be considered as an extension and generalization of fixed point theorems on 2-metric spaces and kannan fixed point theorems, given by many well known authors announced in the available literature.
\end{abstract}

Keywords: Fixed point, Metric Spaces, 2 Metric Spaces, Kannan type mapping, Kannan fixed point theorem.

\section{Introduction And Preliminaries}

The notion of fixed point theorem which may appear as an abstract notion in metric or topological spaces has great importance in applications such as game theory relevant to military, sports and medicine, as well as economics [15]. The concept of a 2-metric space has been initiated and broadly developed by Gähler in a series of papers $[3,4]$ and more. A number of fixed point theorems has been proved for 2-metric spaces. Iseki [11] studied the fixed point theorems in 2-metric spaces. Naidu and Prasad [9] introduced new fixed point theorems on 2- metric space. The study was further enhanced by B.E. Rhoades [7], Miczko and Palezewski [8].Imdad, Kumar and Khan [5] extended their work. After this several researchers used the concept of mappings. Hsiao [16] studied a property of contractive type mappings in 2-metric spaces. Moreover Rhoades [17] and other introduced several properties of 2-metric spaces and proved some fixed point and common fixed point theorems for contractive and expansion mappings and also have found some interesting results in 2-metric space. In [6] the authors introduced compatible mappings, which are more general than commuting and weakly commuting mappings. This concept is useful in obtaining more comprehensive fixed point theorems. Kannan's theorem is independent of the famous Banach contraction principle and that it also characterizes the metric completeness concept. Kannan's result in metric fixed point theory involving contractive type mappings which are not necessarily continuous. It is declared that every kannan type mappings on a complete metric space have unique fixed point. While Subrahmanyam [21] showing that if all the Kannan maps on a metric space have fixed points then that space must necessarily be complete. This is the major reason why kannan type mappings and its generalizations play an important role in Fixed point theory. The concept of kannan type mappings can be seen in a lot of the research works $[10,12,13,14]$.

Definition 1.1 [1, 2]: Let $(\mathrm{X}, \mathrm{d})$ be a metric space and $\mathrm{f}$ be a mapping on $\mathrm{X}$. The mapping $\mathrm{f}$ is called kannan type mapping and has a unique fixed point if there exists $0 \leq \lambda<\frac{1}{2}$ such that $\mathrm{d}(\mathrm{fx}, \mathrm{fy}) \leq \lambda[\mathrm{d}(\mathrm{x}, \mathrm{fx})+\mathrm{d}(\mathrm{y}, \mathrm{fy})] \quad \forall \mathrm{x}, \mathrm{y} \in \mathrm{X}, \lambda \in\left[0, \frac{1}{2}\right)$

A metric space is a set $X$ that has a notion of the distance $d(x, y)$ between every pair of points $x, y \in X$. A metric on a set is a function that satisfies the minimal properties we might except of a distance.

Definition 1.2: A metric $d$ on a set $X$ is a function $d: X \times X \rightarrow[0, \infty)$ such that for all $x, y \in X$ :

i. $\quad d(x, y) \geq 0$ and $d(x, y)=0$ iff $x=y$,

ii. $\quad d(x, y)=d(y, x)$, (symmetry)

iii. $\quad d(x, y) \leq d(x, z)+d(z, y)$ (triangle inequality).

A metric space $(X, d)$ is a set $\mathrm{X}$ with a metric $\mathrm{d}$ defined on $\mathrm{X}$.

We can define many different metrics on the same set, but if the metric on $\mathrm{X}$ is clear from the context, we refer to $\mathrm{X}$ as a metric space and omit explicit mention of the metric $\mathrm{d}$.

Definition 1.3 [20]: Let $X$ be a non empty set. A real valued function $d$ on $X \times X \times X$ is said to be a 2-metric in $X$ if 
i. To each pair of distinct points $\mathrm{x}, \mathrm{y}$ in $\mathrm{X}$. There exists a point $\mathrm{z} \in \mathrm{X}$ such that $\mathrm{d}(\mathrm{x}, \mathrm{y}, \mathrm{z}) \neq 0$

ii. $\quad d(x, y, z)=0$, when at least of $x, y, z$ are equal.

iii. $\quad d(x, y, z)=d(y, z, x)=d(x, z, y)$

iv. $\quad d(x, y z) \leq d(x, y, w)+(x, w, z)+d(w, y, z)$ for all $x, y, z, w \in X$

When $d$ is a 2-metric on $X$, then the ordered pair $(X, d)$ is called 2-metric space.

Definition 1.4: A sequence $\left\{x_{n}\right\}$ in 2-metric space (X, d) is said to be convergent to an element $x \in X$ if $\lim _{n \rightarrow \infty} d\left(x_{n}, x, a\right)=0$ for all a $\in X$.

It follows that if the sequence $\left\{x_{n}\right\}$ converges to $x$ then $\lim _{n \rightarrow \infty} d\left(x_{n}, a, b\right)=d(x, a, b)$ for all $a b \in X$.

Definition 1.5: A sequence $\left\{x_{n}\right\}$ in a 2-metric space $X$ is a Cauchy sequence if $d\left(x_{m}, x_{n}, a\right)=0$ as $m, n \rightarrow \infty$ for all $a \in X$.

Definition 1.6: If a sequence is convergent in a 2-metric space then it is a Cauchy sequence.

Definition 1.7: A 2-metric space (X, d) is said to be complete if every Cauchy sequence in $\mathrm{X}$ is convergent.

Proposition 1.8: If a sequence $\left\{x_{n}\right\}$ in a 2-metric space converges to $x$ then every subsequence of $\left\{x_{n}\right\}$ also converges to the same limit $\mathrm{x}$.

Proposition 1.9: Limit of a sequence in a 2-metric space, if exists, is unique.

Definition 1.10 [23]: Let $(X, d)$ be a metric space. A mapping $T: X \rightarrow X$ is said sequentially convergent if we have, for every sequence $\left\{\mathrm{y}_{\mathrm{n}}\right\}$, if $\left\{\mathrm{T} \mathrm{y}_{\mathrm{n}}\right\}$ is convergence then $\left\{\mathrm{y}_{\mathrm{n}}\right\}$ also is convergence.

Definition 1.11: Let $(X, d)$ be a metric space. A mapping $T: X \rightarrow X$ is said subsequentially convergent if we have, for every sequence $\left\{\mathrm{y}_{\mathrm{n}}\right\}$, if $\left\{\mathrm{T} \mathrm{y}_{\mathrm{n}}\right\}$ is convergence then $\left\{\mathrm{y}_{\mathrm{n}}\right\}$ has a convergent subsequence.

Proposition 1.12: If $(X, d)$ be a compact metric space, then every function $T: X \rightarrow X$ is subsequentially convergent and every continuous function $\mathrm{T}: \mathrm{X} \rightarrow \mathrm{X}$ is sequentially convergent.

Definition 1.13 [22]: A function $\phi: \mathrm{R} \rightarrow \mathrm{R}^{+}$is said to be $\Phi$ function if it satisfies the following conditions

i. $\phi(t)=0$ iff $\mathrm{t}=0$,

ii. $\phi(t)$ is strictly monotone increasing and $\phi(t) \rightarrow \infty$ as $t \rightarrow \infty$,

iii. $\phi$ is left continuous in $(0, \infty)$,

iv. $\phi$ is continuous at 0 .

\section{Main Result}

In this paper, we first studied and have taken the reference of Azam and Arshad [18] who extended the kannan theorem for generalized metric spaces. By taking new extensions of kannan fixed point theorem, we derive some new fixed point theorems and extend some well known results established by Moradi and Alimohammadi [19] for kannan fixed point theorem on complete and generalized metric spaces.

Theorem 2.1: Let $(\mathrm{X}, \mathrm{d})$ be a complete 2-Metric Space, where $\mathrm{f}$ is a self mapping which satisfies the following inequality for all $\mathrm{x}, \mathrm{y}, \mathrm{z} \in \mathrm{X}, \lambda \in[0,1), \mathrm{F} \in \Phi$

$F(d(f x, f y, f z)) \leq \lambda[F(d(x, f x, f x+1))+F(d(y, f y, f y+1))+F(d(z, f z, f z+1))]$

Then $\mathrm{f}$ has a unique fixed point and for every $\mathrm{x}_{0} \in \mathrm{X}$, the sequence of iterates $\left\{\mathrm{x}_{\mathrm{n}}\right\}$ or $\left\{\mathrm{f}^{\mathrm{n}} \mathrm{x}_{0}\right\}$ converges to this fixed point.

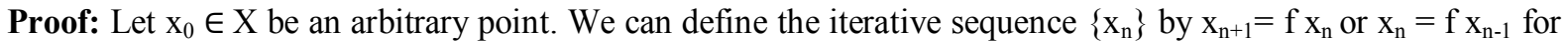
$\mathrm{n} \in \mathrm{N}$.

Now $F\left(d\left(x_{n}, x_{n+1}, x_{n+2}\right)\right)=F\left(d\left(f x_{n-1}, f x_{n}, f x_{n+1}\right)\right)$

$$
\leq \lambda\left[F\left(d\left(x_{n-1}, f x_{n-1}, f x_{n}\right)\right)+F\left(d\left(x_{n}, f x_{n}, f x_{n+1}\right)\right)+F\left(d\left(x_{n+1}, f x_{n+1}, f x_{n+2}\right)\right)\right]
$$

$\leq \lambda\left[\mathrm{F}\left(\mathrm{d}\left(\mathrm{x}_{\mathrm{n}-1}, \mathrm{x}_{\mathrm{n}}, \mathrm{x}_{\mathrm{n}+1}\right)\right)+\mathrm{F}\left(\mathrm{d}\left(\mathrm{x}_{\mathrm{n}}, \mathrm{x}_{\mathrm{n}+1}, \mathrm{x}_{\mathrm{n}+2}\right)\right)+\mathrm{F}\left(\mathrm{d}\left(\mathrm{x}_{\mathrm{n}+1}, \mathrm{x}_{\mathrm{n}+2}, \mathrm{x}_{\mathrm{n}+3}\right)\right]\right.$

In this way

$$
\leq \frac{\lambda}{1-\lambda}\left[\mathrm{F}\left(\mathrm{d}\left(\mathrm{x}_{\mathrm{n}-1}, \mathrm{x}_{\mathrm{n}+1}, \mathrm{x}_{\mathrm{n}+3}\right)\right)\right] \leq\left(\frac{\lambda}{1-\lambda}\right)^{2}\left[\mathrm{~F}\left(\mathrm{~d}\left(\mathrm{x}_{\mathrm{n}-2}, \mathrm{x}_{\mathrm{n}}, \mathrm{x}_{\mathrm{n}+2}\right)\right)\right] \leq \ldots . .\left(\frac{\lambda}{1-\lambda}\right)^{\mathrm{n}}\left[\mathrm{F}\left(\mathrm{d}\left(\mathrm{x}_{0}, \mathrm{x}_{1}, \mathrm{x}_{2}\right)\right)\right]
$$


For every $1, m, n \in N$ such that $1>m>n$, we have

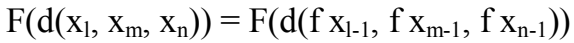

$$
\begin{aligned}
& \leq \lambda\left[\mathrm{F}\left(\mathrm{d}\left(\mathrm{x}_{1-1}, \mathrm{fx}_{\mathrm{l}-1}, \mathrm{f} \mathrm{x}_{1}\right)\right)+\mathrm{F}\left(\mathrm{d}\left(\mathrm{x}_{\mathrm{m}-1}, \mathrm{f} \mathrm{x}_{\mathrm{m}-1}, \mathrm{f} \mathrm{x}_{\mathrm{m}}\right)\right)+\mathrm{F}\left(\mathrm{d}\left(\mathrm{x}_{\mathrm{n}-1}, \mathrm{f}_{\mathrm{n}-1}, \mathrm{fx}_{\mathrm{n}}\right)\right)\right] \\
& \leq \lambda\left[\mathrm{F}\left(\mathrm{d}\left(\mathrm{x}_{\mathrm{l}-1}, \mathrm{x}_{1}, \mathrm{x}_{1+1}\right)\right)+\mathrm{F}\left(\mathrm{d}\left(\mathrm{x}_{\mathrm{m}-1}, \mathrm{x}_{\mathrm{m}}, \mathrm{x}_{\mathrm{m}+1}\right)\right)+\mathrm{F}\left(\mathrm{d}\left(\mathrm{x}_{\mathrm{n}-1}, \mathrm{x}_{\mathrm{n}}, \mathrm{x}_{\mathrm{n}+1}\right)\right]\right. \\
& \leq \lambda\left[\left(\frac{\lambda}{1-\lambda}\right)^{1-1}+\left(\frac{\lambda}{1-\lambda}\right)^{\mathrm{m}-1}+\left(\frac{\lambda}{1-\lambda}\right)^{\mathrm{n}-1}\right] \mathrm{F}\left(\mathrm{d}\left(\mathrm{x}_{0}, \mathrm{x}_{1}, \mathrm{x}_{2}\right)\right)
\end{aligned}
$$

Letting $1, \mathrm{~m}, \mathrm{n} \rightarrow \infty$, we can get

$$
\begin{aligned}
& \lim _{1, m n \rightarrow \infty} F\left(d\left(x_{1}, x_{m}, x_{n}\right)\right)=0 \\
& \lim _{l, m n \rightarrow \infty} d\left(x_{1}, x_{m}, x_{n}\right)=0
\end{aligned}
$$

As $(\mathrm{X}, \mathrm{d})$ be a complete 2-Metric Space, i.e. $\left\{\mathrm{x}_{\mathrm{n}}\right\}$ is a Cauchy sequence and convergent such that $\lim _{\mathrm{n} \rightarrow \infty} \mathrm{x}_{\mathrm{n}}=\mathrm{u}$, where $\mathrm{u} \in \mathrm{X}$.

Hence $F\left(d\left(f u, x_{n+1}, x_{n+2}\right)\right)=F\left(d\left(f u, f x_{n}, f x_{n+1}\right)\right)$

$$
\begin{aligned}
& \leq \lambda\left[F(d(u, f u, f u+1))+F\left(d\left(x_{n}, f x_{n}, f x_{n+1}\right)\right)+F\left(d\left(x_{n+1}, f x_{n+1}, f x_{n+2}\right)\right)\right] \\
& \leq \lambda\left[F(d(u, f u, f u+1))+F\left(d\left(x_{n}, x_{n+1}, x_{n+2}\right)\right)+F\left(d\left(x_{n+1}, x_{n+2}, x_{n+3}\right)\right)\right]
\end{aligned}
$$

Because of the property of $\Phi$ function, $\mathrm{F}$ is continuous, letting $\mathrm{n} \rightarrow \infty$

$\mathrm{F}(\mathrm{d}(\mathrm{f} \mathrm{u}, \mathrm{u}, \mathrm{u})) \leq \lambda[\mathrm{F}(\mathrm{d}(\mathrm{u}, \mathrm{fu}, \mathrm{fu}+1))+\mathrm{F}(0)+\mathrm{F}(0)]$

$F \in \Phi$ so that $F(0)=0$ and therefore $f u=u$.

It is clear that $\mathrm{f}$ has a fixed point.

Theorem 2.2: Let $(X, d)$ be a complete 2-Metric Space, where $f, g$ are self mapping such that $g$ is continuous, one-one and sub sequentially convergent. For all $\mathrm{x}, \mathrm{y}, \mathrm{z} \in \mathrm{X}, \lambda \in[0,1 / 2)$

$d(g f x, g f y, g f z)) \leq \lambda[d(g x, g f x, g f x+1)+d(g y, g f y, g f y+1)+d(g z, g f z, g f z+1)]$

Then $\mathrm{f}$ has a unique fixed point. If $\mathrm{g}$ is sub sequentially convergent then the sequence of iterates $\left\{\mathrm{x}_{\mathrm{n}}\right\}$ or $\left\{\mathrm{f}^{\mathrm{n}} \mathrm{x}_{0}\right\}$ converges to this fixed point.

Proof: Let $\mathrm{x}_{0} \in \mathrm{X}$ be an arbitrary point. We can define the iterative sequence $\left\{\mathrm{x}_{\mathrm{n}}\right\}$ by $\mathrm{x}_{\mathrm{n}+1}=\mathrm{fx}_{\mathrm{n}}$ or $\mathrm{x}_{\mathrm{n}}=\mathrm{fx}_{\mathrm{n}-1}$ for $\mathrm{n} \in \mathrm{N}$.

Now d $\left(\mathrm{g} \mathrm{x}_{\mathrm{n}}, \mathrm{g} \mathrm{x}_{\mathrm{n}+1}, \mathrm{~g} \mathrm{x_{ \textrm {n } + 2 } )}\right) \mathrm{d}\left(\mathrm{g} \mathrm{f}_{\mathrm{n}-1}, \mathrm{~g} f \mathrm{x}_{\mathrm{n}}, \mathrm{g} f \mathrm{x}_{\mathrm{n}+1}\right)$

$\leq \lambda\left[\mathrm{d}\left(\mathrm{g} \mathrm{x}_{\mathrm{n}-1}, \mathrm{~g} \mathrm{f} \mathrm{x}_{\mathrm{n}-1}, \mathrm{gf} \mathrm{x}_{\mathrm{n}}\right)+\mathrm{d}\left(\mathrm{g} \mathrm{x}_{\mathrm{n}}, \mathrm{g} \mathrm{f} \mathrm{x}_{\mathrm{n}}, \mathrm{g} \mathrm{f} \mathrm{x}_{\mathrm{n}+1}\right)+\mathrm{d}\left(\mathrm{g} \mathrm{x}_{\mathrm{n}+1}, \mathrm{~g} \mathrm{fx_{ \textrm {n } + 1 } ,}, \mathrm{g} \mathrm{f} \mathrm{x}_{\mathrm{n}+2}\right)\right]$

$\leq \lambda\left[\mathrm{d}\left(\mathrm{g} \mathrm{x}_{\mathrm{n}-1}, \mathrm{~g} \mathrm{x}_{\mathrm{n}}, \mathrm{g} \mathrm{x}_{\mathrm{n}+1}\right)+\mathrm{d}\left(\mathrm{g}_{\mathrm{n}}, \mathrm{g}_{\mathrm{n}+1}, \mathrm{~g} \mathrm{x}_{\mathrm{n}+2}\right)+\mathrm{d}\left(\mathrm{g} \mathrm{x}_{\mathrm{n}+1}, \mathrm{~g}_{\mathrm{n}+2}, \mathrm{~g} \mathrm{x}_{\mathrm{n}+3}\right)\right]$

Using induction

$$
\left.\leq \frac{\lambda}{1-\lambda}\left[\mathrm{d}\left(\mathrm{g} \mathrm{x}_{\mathrm{n}-1}, \mathrm{~g}_{\mathrm{n}+1}, \mathrm{~g}_{\mathrm{n}+3}\right)\right] \leq\left(\frac{\lambda}{1-\lambda}\right)^{2}\left[\mathrm{~d}\left(\mathrm{~g}_{\mathrm{n}-2}, \mathrm{~g} \mathrm{x}_{\mathrm{n}}, \mathrm{g}_{\mathrm{n}+2}\right)\right] \leq \ldots . . \leq\left(\frac{\lambda}{1-\lambda}\right)^{\mathrm{n}} \mathrm{d}\left(\mathrm{g} \mathrm{x}_{0}, \mathrm{~g} \mathrm{x}_{1}, \mathrm{~g} \mathrm{x}_{2}\right)\right]
$$

For every $1, m, n \in N$ such that $1>m>n$, we have

$$
\begin{aligned}
& \left.\mathrm{d}\left(\mathrm{g}_{\mathrm{x}}, \mathrm{g} \mathrm{x}_{\mathrm{m}}, \mathrm{g} \mathrm{x}_{\mathrm{n}}\right) \leq \lambda\left[\mathrm{d}\left(\mathrm{g} \mathrm{x}_{1}, \mathrm{~g} \mathrm{x}_{\mathrm{m}}, \mathrm{g} \mathrm{x}_{\mathrm{m}-1}\right)+\mathrm{d}\left(\mathrm{g} \mathrm{x}_{\mathrm{m}-1}, \mathrm{~g} \mathrm{x}_{\mathrm{m}-2}, \mathrm{~g} \mathrm{x}_{\mathrm{m}-3}\right)+\ldots .+\mathrm{d}\left(\mathrm{g}_{\mathrm{m}+1}, \mathrm{~g} \mathrm{x}_{\mathrm{m}}, \mathrm{g}_{\mathrm{n}}\right)\right)\right] \\
& \quad \leq\left[\left(\frac{\lambda}{1-\lambda}\right)^{\mathrm{m}-1}+\left(\frac{\lambda}{1-\lambda}\right)^{\mathrm{m}-3}+\ldots .+\left(\frac{\lambda}{1-\lambda}\right)^{\mathrm{n}}\right] \mathrm{d}\left(\mathrm{x}_{0}, \mathrm{x}_{1}, \mathrm{x}_{2}\right) \\
& \quad \leq\left[\left(\frac{\lambda}{1-\lambda}\right)^{\mathrm{n}}+\left(\frac{\lambda}{1-\lambda}\right)^{\mathrm{n}+2}+\left(\frac{\lambda}{1-\lambda}\right)^{\mathrm{n}+4}+\cdots\right] \mathrm{d}\left(\mathrm{x}_{0}, \mathrm{x}_{1}, \mathrm{x}_{2}\right) \\
& \quad \leq\left(\frac{\lambda}{1-\lambda}\right)^{\mathrm{n}}\left[1+\left(\frac{\lambda}{1-\lambda}\right)^{2}+\left(\frac{\lambda}{1-\lambda}\right)^{4}+\cdots\right] \mathrm{d}\left(\mathrm{x}_{0}, \mathrm{x}_{1}, \mathrm{x}_{2}\right) \\
& \quad \leq\left(\frac{\lambda}{1-\lambda}\right)^{\mathrm{n}}\left[\frac{1}{1-\left(\frac{\lambda}{1-\lambda}\right)^{2}}\right] \mathrm{d}\left(\mathrm{x}_{0}, \mathrm{x}_{1}, \mathrm{x}_{2}\right)
\end{aligned}
$$

Since $(\mathrm{X}, \mathrm{d})$ is a complete 2 -metric space and $\left\{\mathrm{x}_{\mathrm{n}}\right\}$ is a cauchy sequence. Where $\mathrm{g}$ is a sub sequentially convergent and $\left\{\mathrm{x}_{\mathrm{n}}\right\}$ has a convergent subsequence. So there exist $\lim _{\mathrm{k} \rightarrow \infty} \mathrm{x}_{\mathrm{n}(\mathrm{k})}=\mathrm{u}$ such that $\mathrm{u} \in \mathrm{X}$ and $\mathrm{k} \in(1$, $\infty)$.

Because $\mathrm{g}$ is continuous, we conclude that $\lim _{\mathrm{k} \rightarrow \infty} \mathrm{g} \mathrm{x}_{\mathrm{n}(\mathrm{k})}=\mathrm{g} \mathrm{u}$. So,

$\mathrm{d}(\mathrm{g} f \mathrm{u}, \mathrm{u}+1, \mathrm{~g} \mathrm{u}) \leq \mathrm{d}\left(\mathrm{g} f \mathrm{u}, \mathrm{x}_{\mathrm{n}(\mathrm{k})}, \mathrm{x}_{\mathrm{n}(\mathrm{k})+1}\right)+\mathrm{d}\left(\mathrm{x}_{\mathrm{n}(\mathrm{k})+1}, \mathrm{x}_{\mathrm{n}(\mathrm{k})+2}, \mathrm{x}_{\mathrm{n}(\mathrm{k})+3}\right)+\mathrm{d}\left(\mathrm{x}_{\mathrm{n}(\mathrm{k})+3}, \mathrm{x}_{\mathrm{n}(\mathrm{k})+4}, \mathrm{~g} \mathrm{u}\right)$ 


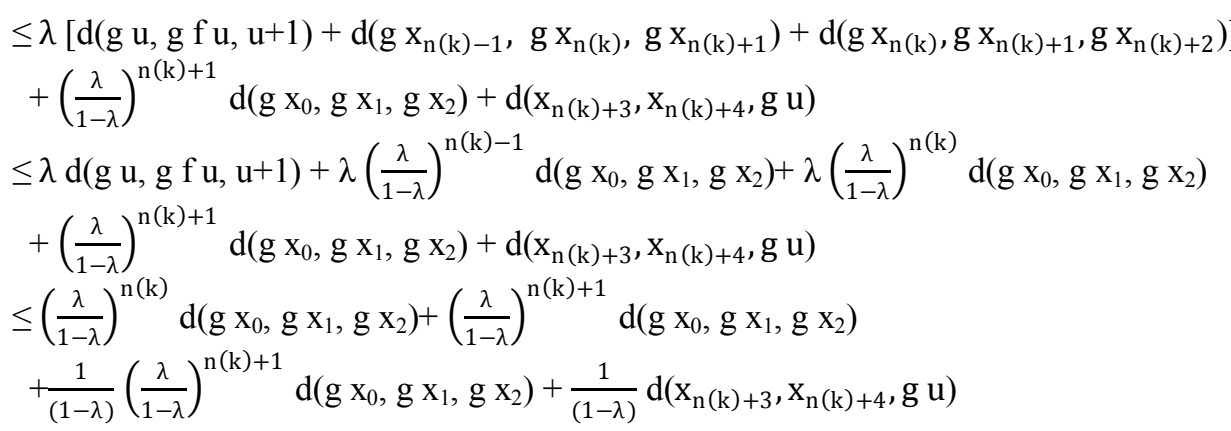

Letting $\mathrm{k} \rightarrow \infty$, we get $\mathrm{d}(\mathrm{g} \mathrm{f} \mathrm{u}, \mathrm{u}+1, \mathrm{~g} \mathrm{u}) \rightarrow 0$.

If $\mathrm{g}$ is sequentially convergent, by replacing $\{\mathrm{n}\}$ with $\{\mathrm{n}(\mathrm{k})\}$, we get $\lim _{\mathrm{n} \rightarrow \infty} \mathrm{x}_{\mathrm{n}}=\mathrm{u}$ and this shows that $\left\{\mathrm{x}_{\mathrm{n}}\right\}$ converges to the fixed point of $\mathrm{f}$. Also, as $\mathrm{g}$ is one-one and $\mathrm{f} u=\mathrm{u}$, we can conclude that $\mathrm{f}$ has a fixed point.

\section{References}

[1]. R. Kannan, Some results on Fixed Point, Bull. Cal. Math. Soc.,60, 1968, 71-76.

[2]. R. Kannan, Some results on Fixed Point II, Amer.Math.Monthly,76, 1969, 405-408.

[3]. S. Gähler, 2-Metrische Räume and ihre topologische strucktur, Math. Nachr., 26, 1963, 115 - 148

[4]. S. Gähler, Uber die unifromisieberkeit 2-metrischer Räume, Math. Nachr., 28, 1965, 235 - 244.

[5]. M . Imdad, M. S. Kumar and M.D. Khan, A Common fixed point theorem in 2-Metric spaces, Math., Japonica, 36(5), 1991, 07-914

[6]. P. P. Murthy, S. S. Chang, Y. J. Cho, B. K. Sharma., Compatible mappings of type (A) and common fixed point theorem, Kyungpook Math. J., 32, 1992, 203-16.

[7]. B. E. Rhoades, Contractive type mappings on a 2-metric space, Math. Nachr., 91, 1979, 151 - 155.

[8]. A. Miczko and B. Palezewski, Common fixed points of contractive type mappings in 2-metric spaces, Math. Nachr., 124, 1985, 341 $-355$

[9]. S.V. R. Naidu and J. R. Prasad, Fixed point theorems in 2-metric spaces, Indian J. Pure Appli. Math., 17(8), 1986, 974-993.

[10]. B. S. Choudhury and K. P. Das, Fixed points of generalized kannan type mappings in generalized menger spaces, Commun. Korean Math. Soc., 24, 2009, 529-537.

[11]. K. Iseki Fixed point theorems in 2-metric spaces, Math Sem Notes, 3, 1975, 133-6.

[12]. O. Kada, T. Suzuki and W. Takahashi, Nonconvex minimization theorems and fixed point theorems in completer metric spaces, Math. Japanica, 44, 1996, 381-391.

[13]. M. Kikkawa and T. Suzuki, Some similarity between contractions and Kannan mappings, Fixed point theory and applications, Article ID 649749, 2008

[14]. P. K Saha and R. Tiwari, An alternative proof of Kannan's fixed point theorem in generalized metric space, News Bull. Cal. Math. Soc., 31, 2008, 15-18.

[15]. K.C. Border, Fixed point theorems with applications to economics and game theory, Coambride University Press, 1990

[16]. A. Hsiao, Property of contractive type mappings in 2-metric spaces, Tnanabha, 16, 1986, 223-39.

[17]. B. E. Rhoades, Contractive type mappings on a 2-metric space, Math. Nachr., 91, 1979, $151-155$

[18]. A. Azam and M. Arshad, Kannan fixed point theorem on generalized metric spaces, J. Nonlinear Sci. Appli., 1, 2008, 45-48.

[19]. S. Moradi and D. Alimohammadi, New extensions of Kannan fixed point theorem on complete metric and generalized metric spaces, Int. J. of Math. Analysis, 5, 2011, 2313-2320.

[20]. R. Tiwari, D. P. Shukla and K. R. Ahirwar, Fixed Point Theorems of Self Mapping in a Complete 2 Metric Spaces, Int. J. Contemp. Math. Sciences, Vol. 7, no. 31, 2012, , $1501-1507$.

[21]. P. V. Subrahmanyam, Completeness and fixed-points, Monatsh. Math., 80, 1975, 325-330.

[22]. B. S. Choudhury and K. P. Das, A new contraction principle in menger spaces, Acta Mathematica Sinica, English Series, 24, 2008, 1379-1386.

[23]. A. Beiranvand, S. Moradi, M. Omid and H. Pazandeh, Two Fixed-Point Theorems For Special Mappings, Math. F A, 2009. 